\title{
Painful legs and moving toes: evidence on the site of the lesion
}

\author{
P. W. N A T H A N ${ }^{1}$ \\ From the National Hospital for Nervous Diseases, Queen Square, London
}

SUMMARY A condition of painful legs with moving toes was described in 1971. Further examples of this condition are now reported, showing lesions in the posterior root ganglion, cauda equina, nerve roots, or a peripheral nerve of the lower limb. It is concluded that this syndrome is caused by a lesion of the afferent fibres of the posterior nerve roots. It is likely that this lesion causes frequent spontaneous impulses in the posterior roots which activate local circuits of interneurones and motoneurones and result in co-ordinated movements involving local muscles.

The condition of painful legs with moving toes described by Spillane et al. (1971) has remained unexplained. However, in their paper, they drew attention to the fact that three of the six patients reported had a history of lumbago and sciatica and "had evidence of damage to the lower lumbar intervertebral discs; and in two of the patients in whom a biopsy sample of a peripheral nerve was examined, there were some degenerated nerve fibres."

The main purpose of this paper is to present some further cases to show that the lesion causing the syndrome is likely to be in the peripheral nerve, posterior root ganglion, or the cauda equina. It is likely that impulses are generated in these structures and pass into the spinal cord, some to higher regions to cause pain, others into the local interneurone pool, and thence to motoneurones, to produce the co-ordinated movements of the toes.

\section{Case reports}

CASE 1

Mrs BH, aged 46 years, had herpes zoster in May 1973. The skin lesions were on a strip along the outside of the right thigh, to a lesser extent on the outside of the leg to the ankle and in a small

\footnotetext{
${ }^{1}$ Member of the External Scientific Staff of the Medical Research Council.

Address for reprint requests: The National Hospital for Nervous Diseases, Queen Square, London WC1N 3BG.

Accepted 19 May 1978
}

patch on the medial side of the thigh. It was "terribly painful for two or three weeks." Aftes 0 that time the skin became sensitive so that "on $\underset{\perp}{\omega}$ cannot bear to have clothes on it," and she fount herself moving away from people in case the touched her.

At some point in 1975 , the toes of the right foo started moving spontaneously. It was mostly side to-side movements of the four lateral toes. She could stop them by extending her toes strongly? but as soon as she stopped doing this, the movements returned. Although the movements were themselves painless, she said: "the wiggling of my toes can drive me mad; a ceaseless movement." She had even tried tying them up in a bandage to stop them moving. If this did succeed at all, the movements were worse when the bandage was removed.

Examination in January 1978 showed minimal scarring of the skin from the herpes. The jerks were normal and equal, and there was no weakness and no loss of sensibility.

From the few scars present and the description of her pain and scarring, we deduced that the original herpes affected the fifth lumbar ganglion. The distribution was that described by Keegan and Garrett (1948). In addition, the scarring on the medial side of the thigh was thought to be in the territory of the cutaneous branch of the obturator nerve.

Dr N. M. F. Murray was kind enough to perform nerve conduction studies of the lower limbs. The latency of the $\mathrm{H}$ reflex of the two limbs was normal and equal. The motor nerve conduction velocities of 
both lateral popliteal and posterior tibial nerves were normal, as were the muscle action potentials. The sensory action potentials of both sural nerves were of normal amplitude and latency. There were frequent discharges of single motor units of the abductor digiti minimi on the right. Dr Murray considered that "the large units in this muscle suggest chronic partial denervation."

Comment In this disorder, the virus causes destruction in the posterior root ganglion, and it also frequently spreads into the posterior horn. From there it may be spread to the anterior horn and up and down the cord for about one segment. In this case, the sensory abnormalities incriminated the fifth lumbar ganglion, and the abnormal movements the first sacral motoneurones.

The movements of the toes came on about two years after the lesion. This long interval is typical of all the cases. In none of the patients did movements start as soon as the damage to the lumbosacral roots occurred. This case was not typical in that pain was not an outstanding symptom; although the skin was sore and hypersensitive, the patient did not complain of constant pain.

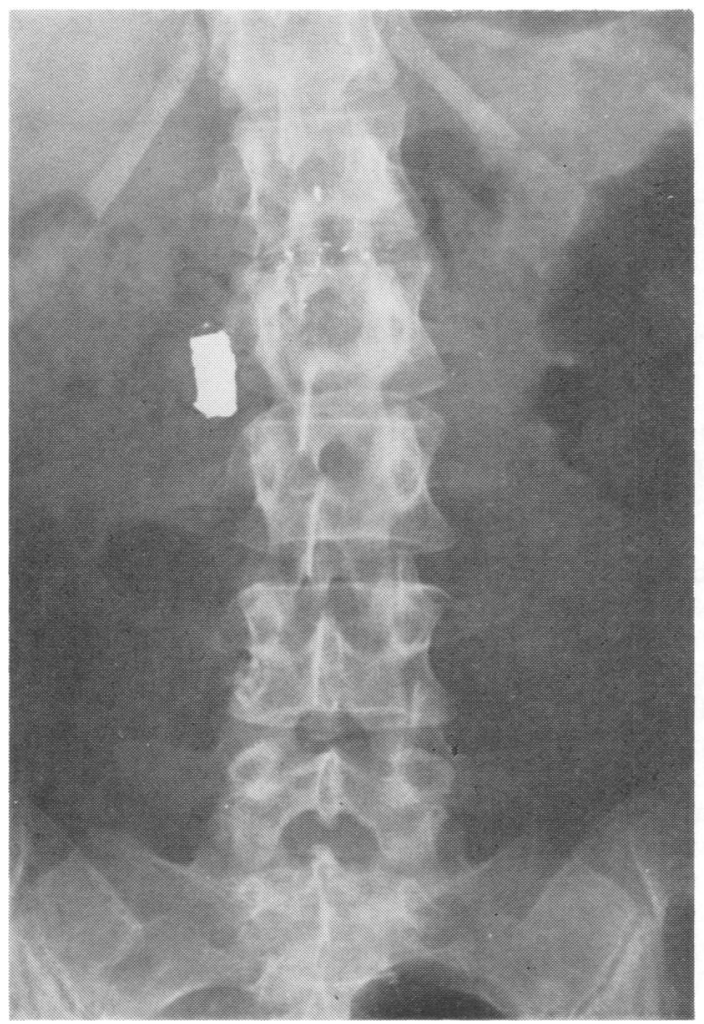

Figure Radiographs to show bullet behind the 1st and 2nd lumbar vertebrae on right.

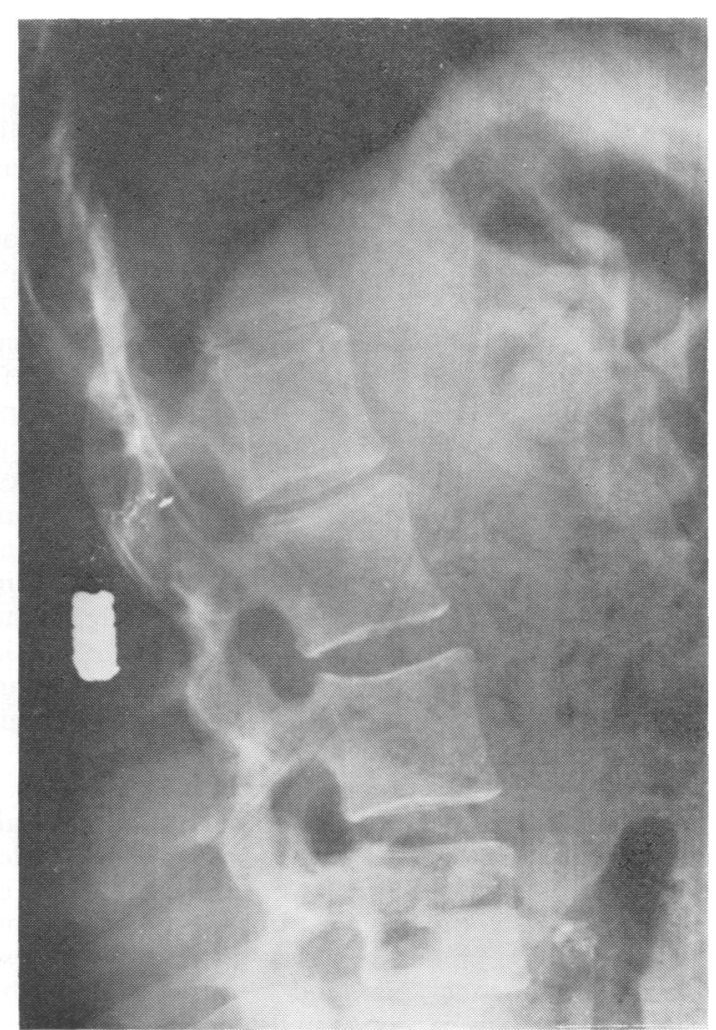

CASE 2

For the excellent notes of the early history of case 2, I am grateful to Professor J. Gonzalez Cruchaga of the Hospital del Salvador in Santiago, Chile.

Mrs SB, an air hostess, aged 30 years, was shot in 1970 by a hi-jacker. The bullet entered the ninth intercostal space in the left midaxillary line. She immediately fell to the ground, unable to move her legs. She was admitted to hospital within half an hour, given a blood transfusion, and operated upon. The bullet had pierced the spleen and pancreas and several intestinal loops; it came to lodge behind the first and second lumbar vertebrae on the right, where it can be seen in the Figure.

From the moment the patient was admitted to hospital, she had intense pain in the lower limbs, and pressure on the deep tissues was painful. A diagnosis of a lesion of the cauda equina was made. After the operation on the abdomen, the patient still continued to suffer from severe pain in both lower limbs, aggravated by any contact. Apart from this pain when her legs were touched, she felt numb and dead below the waist, and she 
could not move either lower limb. Examination revealed a flaccid paraplegia with loss of deep and superficial reflexes, including the plantar responses. There was retention of urine and faeces. There was absence of all forms of sensibility below the waist, though heavy pressure or moving the limbs passively caused pain. Professor Gonzalez Cruchaga wrote to us in 1977: "there followed a slow but steady improvement. The neuropathic bladder recovered, and after several weeks it was possible to remove the catheter and start bladder training. The motor power recovered in several months but there is still weakness of the distal muscles of the left leg, with atrophy. Reflexes reappeared. The sensory deficit cleared slowly and at some time there was a radicular type of anaesthesia mixed with hyperalgesia."

She was seen nine months after the injury by Dr Morris Bender in New York, who considered that the diagnosis was a concussion of the lumbar roots. She adapted well to a life of being an invalid in pain; she married, and had a baby. By 1970 she had begun to walk again, but was impeded by painful cramp in the left leg. Very frequently, and particularly when the weather was cold and when she was emotionally tense, the left foot would plantarflex and invert for periods of a half to one and a half minutes. The relevant muscles were in cramp and this was very painful. It was probably at this time that the spontaneous small movements of the toes started.

In November 1977, she was sent to see Professor W. Ian McDonald at this hospital. Through his kindness I saw the patient on 24 November 1977. She came to London seeking help for continuing severe pain and abnormal movements of the left lower limb. She told us that she had a constant deep ache on which was superimposed every few minutes, a strong muscle spasm with a burst of increased pain. This was the pain that normal people get if their muscles go into a ball of contraction with cramp. The constant pain was in the left foot, leg, and back of the thigh, and sometimes in the knee. The maximal pain would come on when the muscles went into spontaneous spasm, flexing and abducting the left toes. This would last up to half a minute.

There were ceaseless movements of the toes of the left foot. The movements were mainly small flexions and extensions with a minimum of sideto-side movements. There were also slight movements of the foot. She was unable to stop them. Several of the muscle spasms were seen. These were spontaneous, maximal contractions of the muscles that were continually performing small movements, moving the foot and toes in flexion and extension and abduction of the big toe.

When she walked she did so slowly, with slapping of the left foot. The left quadriceps, tibialis anterior, and small muscles of the foot were wasted. Deep reflexes were absent in both lower limbs; the right plantar response was equivocal, the left was normal. There were trophic changes on the sole of the left foot. There was reduced sensibility to all forms of stimulation on the left from L1 to S5 and on the right from L5 to S5 dermatomes. Throughout the regions of diminished sensibility, there was a normal flare on scratching with a pin. Conduction velocity in both sural nerves was normal.

We concluded that some metallic fragments, seen in the Figure, were within the spinal canal. A metrizamide radiculomyelogram was carried out. The contrast material passed easily throughout the affected region, and there was only a small vertical defect on the left lateral aspect suggesting a local thickening of a nerve root.

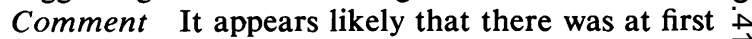
a concussion of the whole of the lower spinal cord, from the level of the T10-11 segmenp? caudally. There is now a permanent lesion of the cauda equina, partially involving the $L 1$ to $S$. roots on the left and the L5 to S5 roots on the right. There is a partial lesion of the relevand $\overrightarrow{0}$ nerve roots causing pain and moving toes. The movements differ from most of our other cases io 웅 that they are mainly flexion and extension, an $\mathbb{Q}$ these movements are better co-ordinated than $\overrightarrow{0}$ usually the case. The same muscles as were constantly contracting and relaxing went into spasms with, apparently, all available motor units firing.

CASE 3

Dr IM, aged 57 years, was referred to me by $\operatorname{Dr} \frac{\mathscr{Q}}{\mathbb{Q}}$ John Walsh from Sydney, on account of attacks $\varrho$ of severe pain in the right leg and foot associated with fasciculations in the extensor digitorum $\exists$ brevis and marked vascular abnormalities of the skin. Dr IM related that three years previously he had spontaneously developed pain in the right big toe and heel. The pain felt at first as though $\overline{0}$ he had cut himself on a piece of glass. As the pain got worse, the skin of the big toe and neighbouring region of the foot was always of an $\delta$ abnormal colour and vascularity, either blue or $₹$ white or vasodilated and red. About 18 months 옹 after the onset, he was given beta-blockers for $>$ hypertension. These drugs aggravated the pain so much that he would lie on the floor screaming. N When the region was dilated and very red, the $\mathrm{N}$ pain was a severe burning, as if someone were $N$ burning the region with a blow-lamp. The pain was 
relieved by standing. He had had to give up practising as a doctor.

About one year after the onset of the pain, he saw that his toes were moving spontaneously. He could stop this for a minute or two but as soon as he ceased trying to stop it, the movements would return. When the toes were moving, there was a pain over the foot but not the heel-a pain as though it were being slashed with razor blades.

Shortly after the movements began, a myelogram was carried out and showed a cyst. At operation a large Tarlov cyst was found, compressing the right first sacral root. "The cyst was decompressed and the stretched and compressed sensory nerve root was severed."

This operation relieved the pain in the leg but not in the foot where it continued to get worse. It was related to the vascular state-when the vessels of the skin were dilated and the foot was red, the pain was excruciating. The operation had no effect on the spontaneous movements.

When I saw the patient in April 1976, there were minimal movements of the left big toe and vasodilatation of the skin. On the right, there was vasodilatation over most of the foot, the long flexors and extensor of the hallux and digits were showing intermittent contractions and extensions, and the toes were showing the typical movements of uneven flexion, extension, and adduction and abduction. The knee jerks were present, ankle jerks absent. Pressure on the top of the fibula and over the lateral popliteal nerve sent shooting pains down to the dorsum of the foot and under the heel.

Comment In this case the typical pain and moving toes were associated with a cyst compressing the first sacral root. Unusual were the erythromelalgia and the increase of the pain associated with beta-blocking drugs.

CASE 4

Mrs SL, aged 62 years, whom I saw in March 1974, had fallen down a flight of stairs in November 1971, cutting the skin deeply at the junction of the ankle and the dorsum of the foot. She was taken to hospital and the wound was closed with 10 stitches.

As soon as she started walking about again, she got "a numbed prickly feeling in the skin of the foot, like electric shocks." Since that time it has become worse. It is worse when she rests, it wakes her from sleep, and is relieved by walking. She said that pain was perhaps not the correct description-better was "a sickly feeling, a numbness with electricity as well." She noted between 1974 and 1976 that the lateral four toes of the foot had started moving spontaneously. These movements accompanied the pain, though they did not worry or upset her. They were more marked when she was sitting or lying, and were increased immediately after she tried to stop them. For the first year or so she was able to stop the movements by moving the toes up and down. Eventually it became harder to stop them, and she could do so only by tensing all muscles of the ankle and foot. She said that even when the movements were not very marked "it feels like a moving mass insideand also like someone standing on the centre of my foot." Examination showed the typical moving toes without any movements of the big toe. In the terminal distribution of the musculocutaneous nerve, which had clearly been divided by the cut on the dorsum of the ankle, some touches with cottonwool were not felt. Pinprick was felt but diminished, though at some points it hurt more than in normal areas. All stimuli felt "tinglingelectric." It was noted that this region of abnormal sensibility extended $10-20 \mathrm{~mm}$ proximal to the scar. The scar itself was no more sensitive than the surrounding region.

Comment This patient got pain and moving toes from a peripheral nerve lesion. The pain was not severe.

Since my colleagues and I described this condition in 1971 I have seen other patients suffering from it, in addition to those described here. Most but not all of them had had some slight or severe lesion affecting the lumbar roots, though they had not all had backache. Some of them had had one or more surgical interventions on prolapsed intervertebral discs followed by chronic pain. In these cases a diagnosis of arachnoiditis had usually been made, confirmed at operation or by myelography. After several years the movements of the toes appeared. One patient had never noticed the movements himself but his wife had seen them during his sleep. Another patient had arachnoiditis after surgery for herniated disc. He had had an episode of moving toes lasting for some months. This had then stopped but the pain remained. He was the only patient in whom the moving toes part of the syndrome went away.

It may be that occasionally similar pain and spontaneous movements occur in other muscles. For instance we have seen a patient with a painful lesion of the ilioinguinal nerve and spontaneous slow contractions and relaxations of the dartos muscle.

\section{Discussion}

The curious combination of severe pains in the legs 
and feet and spontaneously moving toes was described in 1971 by Spillane et al. Evidence is now presented to indicate that the discharging lesion causing both the pain and the moving toes is in the posterior root ganglion or posterior nerve root or in afferent fibres or peripheral nerves.

If, at operation on the human spinal cord, the posterior roots within the lumbar or the cervical region are stimulated, large, co-ordinated movements of the limb are induced involving many muscles. The movements of moving toes are similar. They are properly co-ordinated movements, involving several muscles-they include the long flexors and extensors of the toes. I would suggest that these movements are caused by posterior root fibres firing frequently and spontaneously, and that this input to the local segments of the spinal cord induces the firing of related motoneurones. The circuit from the posterior roots to the motoneurones includes the interneurones necessary to organise reciprocal innervation and correct timing.

These movements are unlike those that originate in motoneurones. Such movements are seen in the face as facial myokymia, illustrated electromyographically by Hjorth and Willison (1973). The movements caused by motor nerve fibres firing spontaneously are different. They are seen with overbreathing combined with ischaemia of a peripheral motor nerve, and in the post-ischaemic period. During ischaemia with overbreathing there are quivering fasciculations of groups of muscle fibres. In the post-ischaemic phase, there is firing of many or all motor fibres and this causes a contraction of the muscle fibres supplied. All muscle fibres contract, producing a contraction like that of night cramps. The small, creeping, changing movements of moving toes are unlike these varieties of movements. Since they do not arise in motor fibres nor in spontaneously firing motoneurones, it can be deduced that both the pain and the movements arise in the posterior root fibres or ganglia. It must be pointed out, however, that not all the patients have shown any pathological condition affecting these structures.

Further experience in seeing patients with pain and moving toes has led to some further knowledge about the syndrome. The pain usually starts before the movements. For example, one patient had constant unexplained deep pain in the leg and foot. Electromyographic examination showed single motor units firing occasionally and spontaneously, just as in normal people. But as months went by motor units would fire more frequently. Then several motor units of several muscles would fire, until at the end of two to three years, motor units of short and long flexors and extensors, $\stackrel{\mathbb{D}}{=}$ adductors, and abductors of the toes would fire $\bar{\circ}$ in the typical co-ordinated small movements of $\stackrel{\text { }}{=}$ moving toes. An unusual case, perhaps different ${ }^{\circ}$ from the others, was Mrs SB (case 2). In her case ${ }^{0}$ the small flexions and extensions of the toes would $\widehat{c}$ increase until there was a strong contraction of the limb induced by most of the flexors contracting fully. The muscles were strongly contracted, resembling the cramps that occur in normal $\stackrel{0}{+}$ people.

These patients are presented not merely for $\frac{\bar{\sigma}}{\bar{n}}$ their clinical interest, but also as examples of $\frac{\bar{D}}{\vec{D}}$ movements generated in the posterior root fibres $\stackrel{\mathbb{Q}}{2}$ or ganglia. It is proposed that the co-ordinated movements of the toes are caused by the input from posterior root fibres exciting local spinal interneurones. The spontaneous firing can be $\vec{\omega}$ stopped by tracts mediating conscious control, but $\stackrel{\sigma}{\circ}$ as soon as the conscious inhibition ceases, the local circuits regain their autonomous activity.

The syndrome has not been described in the $\nexists$ upper limbs although there are hints of minimal $\overrightarrow{0}$ parts of it appearing. Patients with lesions affecting 8 the cervical roots complain of constant numbnes $\stackrel{\omega}{\oplus}$ and painful tingling or pins and needles, anf의 음 sometimes describe occasional flickerings of the interossei or abductor of the little finger.

If ever the pain stops, the movements stop too But unfortunately nothing new has been found alleviate the pain; and the pain may be severe Blocking the sympathetic supply to the lower limb may stop the pain and movements, but only temporarily. In one patient, we blocked the lumbar sympathetic chain with aqueous phenol solution. It stopped the pain and movements for 30 hours; then both returned, although the sympathetic block remained effective. In our previous paper (Spillane et al., 1971) we suggested some relationship between the pain and movements and the sympathetic system. My further experience has shown that blocking the sympathetic supply has a non-specific effect on the pain of those patients-just as it has in many painful conditions. There is no particular relationship between the sympathetic nerves and this syndrome. This statement can be made with assurance for the syndrome has been seen to affect one patient who had already had a sympathectomy for vascular occlusion of the lower limbs.

\section{References}

Hjorth, R. J., and Willison, R. G. (1973). The electromyogram in facial myokymia and hemifacial spasm. Journal of the Neurological Sciences, 20, 117-126. 
Keegan, J. J., and Garrett, F. D. (1948). The segmental distribution of the cutaneous nerves in the limbs of man. Anatomical Record, 102, 409-438.
Spillane, J. D., Nathan, P. W., Kelly, R. E., and Marsden, C. D. (1971). Painful legs and moving toes. Brain, 94, 541-556. 